\title{
Relation between iridopathy and retinopathy in diabetes
}

Francesco Bandello, Rosario Brancato, Rosangela Lattanzio, Marcello Galdini, Bruno Falcomatà

\begin{abstract}
In order to assess the relation between diabetic iridopathy (DI) and retinopathy (DR), 225 eyes of 117 diabetics with clear media were evaluated. Each patient underwent iris and retinal fluorescein angiography, which was used to classify DI and DR. DI was classified as: absence of DI; non-proliferative DI; proliferative DI; neovascular glaucoma. DR was classified as: absence of $D R$; background $D R$; pre-proliferative DR; proliferative DR. The sensitivity of iris fluorescein angiography in assessing DR was $44.5 \%$, the specificity $88 \%$, the positive predictive value $92.8 \%$, and the negative value $31 \cdot 2 \%$. In pre-proliferative and proliferative DR, fluoroiridographic detection of iris neovessels gave a sensitivity of $56 \%$ and a specificity of $100 \%$. The positive predictive value was $100 \%$ and the negative value $65 \%$. In conclusion, iris fluorescein angiography yields valuable information on $D R$ and is a helpful basis for avoiding complications when scheduling eyes with dioptric media opacities for surgery.
\end{abstract}

(Brf Ophthalmol 1994; 78: 542-545)

In diabetic patients the whole vascular system of the eye can be affected by microangiopathy; iridopathy and retinopathy are two of its most severe manifestations. ${ }^{1}$

The presence of advanced forms of diabetic retinopathy (DR) and/or diabetic iridopathy (DI) contraindicates many ophthalmic surgical procedures. The high level of neovascular complications in diabetic patients is well known, as is the higher risk of neovascular glaucoma following vitrectomy ${ }^{2-6}$ and cataract extraction, ${ }^{7-17}$ particularly intracapsular extraction. ${ }^{18}$ This means that abnormalities of the iris and retina must be assessed carefully before any surgical manipulation in order to avoid complications. Sometimes, however, DR is not detectable because of opacity of the dioptric media. In these cases, iris fluorescein angiography is the only way to evaluate diabetic microangiopathy and hence to make an indirect assessment of the DR. ${ }^{19-22}$

To date, the relation between iridopathy and retinopathy in diabetes has not been clearly elucidated. The aim of this study was to assess the relation between detectable iris and retinal lesions in diabetic patients without dioptric media opacities in order to answer the following questions: (1) When DI is absent, what type of DR will be present? (2) When non-proliferative DI is present, what type of retinopathy exists with it? (3) When proliferative DI is present,

\begin{tabular}{ll}
$\begin{array}{l}\text { Table } 1 \text { Clinical characteristics of patients included in the } \\
\text { study }\end{array}$ \\
\hline Age (years) (SD) & $58 \cdot 0(13 \cdot 9)$ \\
Sex & $53(45 \cdot 3 \%)$ Males \\
Diabetes mellitus & $48(41 \%)$ Insulin dependent \\
Type & $15 \cdot 8(9 \cdot 3)$ \\
Duration (years) (SD) & $0 \cdot 61(0 \cdot 33)$ \\
Visual acuity (SD) & \\
\hline
\end{tabular}

what type of retinopathy exists with it? And therefore: (4) Is DI an indicator of DR?

\section{Materials and methods}

Patients were recruited from among all those who visited our outpatient department between January and April 1991. The inclusion criteria were diabetes mellitus type I or type II. The exclusion criteria were: opacities of the dioptric media, causing difficulties in retinal fluorescein angiography; other ocular diseases; topical myotic therapy; previous laser treatment; previous ocular surgery.

A total of 225 eyes of 117 patients were included in the study. Nine eyes were excluded; three because of opacities of the dioptric media, two because of retinal vein occlusion, and four because of previous laser treatment. The clinical characteristics of all patients included in the study are summarised in Table 1 . They all provided informed consent for entry to the trial.

All patients underwent a complete ocular examination, a fluorescein angiography of the iris, and stereoscopic colour fundus photographs of seven standard fields. ${ }^{23}$ Iris fluorescein angiography was performed using a modified standard optics Zeiss photo slit-lamp, with a dual filter system (excitation filter and barrier filter) and the

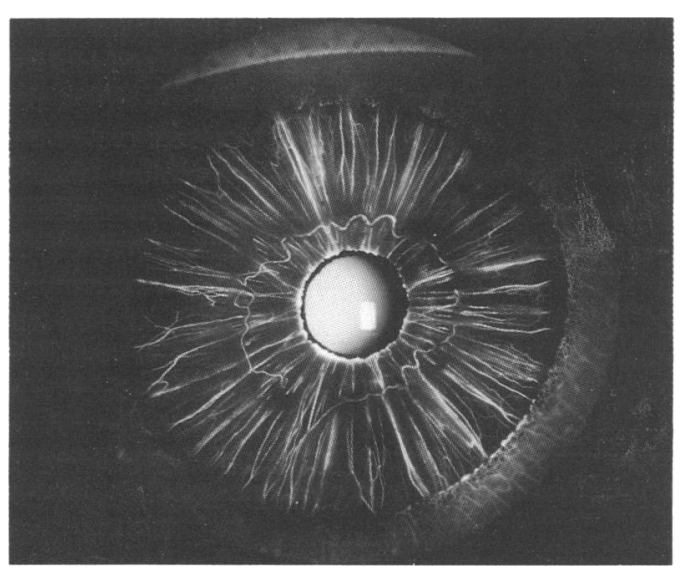

Figure 1 Iris fluoroangiographic picture of a diabetic patient without iridopathy; the morphology and permeability of iris vessels are within the normal range, and no dye leakage is present even though this is a late phase angiogram. 
Figure 2 Fluorescein angiographic image of nonproliferative diabetic iridopathy with dye leakage at the pupillary margin and also at the stromal vessels (late phase).

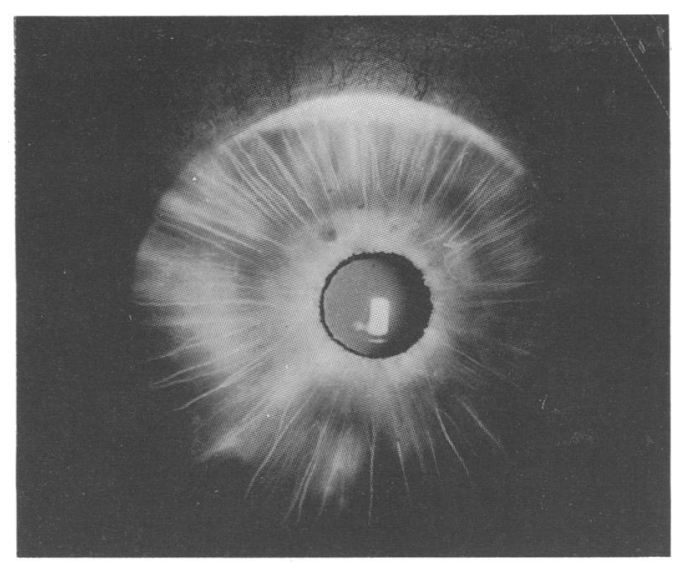

Figure 3 Angiographic findings in a case of proliferative diabetic iridopathy with new vessels over the whole surface of the iris.

Figure 4 Iris fluorescein angiography in a 71-year-old diabetic subject with dye leakage at the pupillary border.
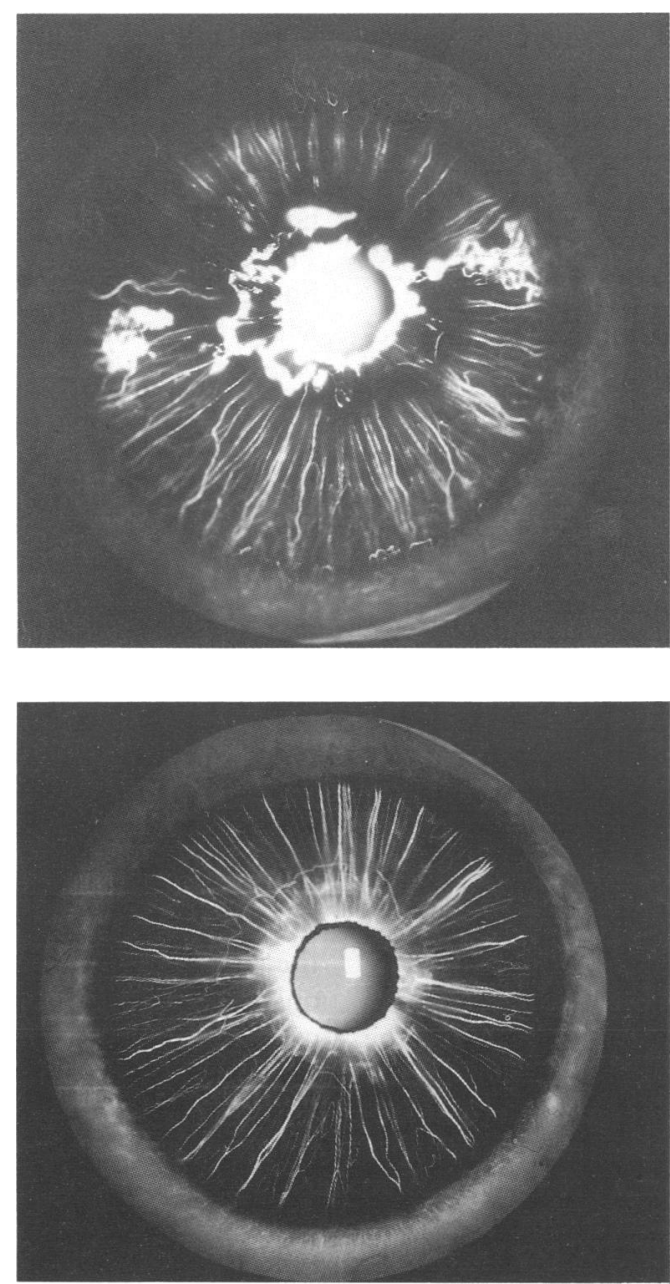

accessories necessary to photograph the anterior segment of the eye. At the start of the examination, fluorescein was injected intravenously (as in retinal angiography). A $5 \mathrm{ml}$ dose of a $20 \%$ solution of fluorescein was used (about $14 \mathrm{mg} / \mathrm{kg}$ body weight); care was taken that pupils were not dilated. Then, after at least 48 hours but within 2 weeks from iris fluorescein angiography, a retinal fluorescein angiography was performed. Fundus photographs were graded by one of us (MG) using an abbreviation of the Modified Airlie House classification. ${ }^{23}$ Blind retinal and iris angiogram evaluations were carried out separately by two of us (RL, BF) specialised in these fields. Each eye was then classified according to the degree of DI and DR found. Retinal angiograms were employed in DR classification to confirm the gradings assessed by colour fundus photographs. The iris and retinal photographs were then matched.

DI was classified as follows:

grade 0: absence of DI (no DI) (Fig 1); fluorescein leakage is absent in late phases too; grade 1: non-proliferative DI (NPDI), with dilated pupillary and stromal capillaries which let the dye leak through, giving rise to slight, short lasting fluorescence. Once the dye bolus has passed through, the hyperfluorescence tends to diminish and disappear, a diffuse veil remaining in the anterior chamber (Fig 2);

grade 2: proliferative DI (PDI), with new vessels at the pupillary margin and/or stroma, filling rapidly with dye and leaking equally promptly and diffusely (Fig 3);

grade 3: neovascular glaucoma (NVG), with newly formed fibrovascular tissue on the iris surface and at the iridocorneal angle, associated with intraocular hypertension.

Pupillary margin leakage was considered physiological in patients aged over 50 years $^{2426}$; therefore, eyes with age-related pupillary margin leakage only were classified in the group without DI (Fig 4).

Figures 1-4 were used as the standard angiograms against which all eyes were compared in order to minimise individual variations within each classification.

DR was classified as follows:

grade 0: absence of DR (no DR), corresponding to level 1 of the abbreviated version of the Modified Airlie House classification ${ }^{23}$;

grade 1: background DR (BDR), comprising levels 2, 3, and 4;

grade 2: pre-proliferative DR (PPDR), corresponding to level 5;

grade 3: proliferative DR (PDR), equivalent to level 6.

\section{Results}

Table 2 compares the DI and DR group findings. In the 141 eyes without DI, $31 \%$ had no DR, $34 \%$ had BDR, $21 \%$ had PPDR, and $14 \%$ had PDR (question 1).

In the 14 eyes with NPDI almost half had no accompanying DR, $15 \%$ had BDR, $21 \%$ had PPDR, and the remaining $21 \%$ had PDR (question 2). The mean age distribution of the patients with NPDI was: (1) patients without DR: 52.8 years (SD 15.4); (2) patients with BDR: $57 \cdot 4$ years (SD 11.7); (3) patients with PPDR: $45 \cdot 4$ years (SD 13.2); and patients with PDR: $62 \cdot 5$ years (SD 21.7).

The 68 eyes with PDI all had severe retinopathy; it was pre-proliferative in one third and

Table 2 Relation (\%) between diabetic iridopathy (DI) groups and diabetic retinopathy $(D R)$ groups

\begin{tabular}{lccclc}
\hline & NoDI & NPDI & PDI & NVG & Total \\
\hline No DR & $44(31)$ & $6(43)$ & 0 & 0 & $50(22)$ \\
BDR & $48(34)$ & $2(15)$ & 0 & 0 & $50(22)$ \\
PPDR & $29(21)$ & $3(21)$ & $25(37)$ & 0 & $57(26)$ \\
PDR & $20(14)$ & $3(21)$ & $43(63)$ & $2(100)$ & $68(30)$ \\
Total & 141 & 14 & 68 & 2 & 225 \\
\hline
\end{tabular}

No $D R=$ absence of $D R ; B D R=$ background $D R ; P P D R=$ pre-

proliferative DR; PDR = proliferative DR.
No DI = absence of DI; NPDI = non-proliferative DI;

$\mathrm{PDI}=$ proliferative DI; NVG= neovascular glaucoma. 
Table 3 Comparison (\%) of mild and serious forms of diabetic iridopathy $(D I)$ and diabetic retinopathy $(D R)$

\begin{tabular}{llll}
\hline & NoDI/NPDI & PDI/NVG & Total \\
\hline No DR/BDR & $100(65)$ & 0 & $100(44)$ \\
PPDR/PDR & $55(35)$ & $70(100)$ & $125(56)$ \\
Total & 155 & 70 & 225 \\
\hline
\end{tabular}

No $D R=$ absence of $D R ; B D R=$ background $D R ; P P D R=$ pre proliferative DR; $P D R=$ proliferative DR.

proliferative DR; $P D R=$ proliferative $D R$.
No DI = absence of DI; NPDI = non-proliferative DI;

$\mathrm{PDI}=$ proliferative $\mathrm{DI} ; \mathrm{NVG}=$ neovascular glaucoma.

proliferative in two thirds. The two cases of NVG both had concomitant PDR (question 3). In answer to question 4: Is DI an indicator of DR?, and thus to assess whether fluoroiridographic detection of DI is useful as an indirect assessment of DR, we considered:

- patients without DI and without DR as true negative;

- patients with DI and DR as true positive;

- patients without DI but with DR in any form as false negative;

- patients with DI in any form but with no fluoroangiographic retinal lesions as false positive.

The specificity of fluoroangiographic detection of DI in assessing DR turned out to be $88 \%$ and the sensitivity was $44 \cdot 5 \%$. The positive predictive value was $92 \cdot 8 \%$, the negative value was $31 \cdot 2 \%$.

In clinical practice it is helpful to detect serious forms of DR before scheduling cararact or retinovitreal surgery, in order to avoid complications. Table 3 summarises the picture we obtained when we related the presence or absence of serious DI (PDI and NVG) to the presence or absence of serious DR (PPDR and PDR).

Fluoroiridographic detection of iris neovessels turned out to have a specificity of $100 \%$ in assessing the serious forms of $\mathrm{DR}$, and its sensitivity was $56 \%$. The positive predictive value was $100 \%$ and the negative value $65 \%$. In the case list, therefore, all the eyes with iris neovessels had PPDR or PDR, and $56 \%$ of the eyes with serious DR had iris neovessels.

\section{Discussion}

In diabetic patients it is important to be able to establish what type of retinopathy is behind a cataract or other type of dioptric media opacity, as serious complications can develop in a patient operated on without this knowledge. ${ }^{2-18}$

For some time now we have been using iris fluorescein angiography in the preoperative evaluation of diabetic patients, as we are led to believe that the degree of iridopathy present is indicative of the degree of retinal microangiopathy. Many studies have been published on iris fluorescein angiography in diabetic patients, ${ }^{27-34}$ but only one other study (by Algvere and Kornacki ${ }^{19}$ ) evaluates the relation between DR and DI, using both iris and retinal fluoroangiography. Our results basically confirm those of Algvere and Kornacki, even though they used a different method of classification of DI and DR.

Iris fluorescein angiography was certainly a helpful diagnostic tool, because the detection of DI in any form (NPDI, PDI, NVG) was associated with DR in all but $7 \%$ of the cases in our study. Furthermore, each time proliferative iridopathy was detected, the patient also had serious pre-proliferative or proliferative DR. Clinically therefore, when iris neovascularisation is detected in an eye in which it is impossible to evaluate retinopathy because of opacity in the dioptric media, concomitant serious retinopathy must be presumed, and great care must be taken in the management of the eye. Before cataract extraction or vitrectomy, cryocoagulation ${ }^{21}$ or transscleral photocoagulation ${ }^{35}$ must be done to destroy the non-perfused peripheral areas of the retina. Sometimes endophotocoagulation carried out in the course of vitrectomy ${ }^{36}$ or photocoagulation done in the days immediately after cataract extraction can produce just as good results. ${ }^{21}$

Moreover, iris fluorescein angiography should not be seen as an invasive diagnostic procedure which is without value. It would be a mistake to think that even a well carried out biomicroscopic examination can provide the same level of diagnostic information. In a study we carried out on 114 eyes of 63 diabetic patients we used iris fluorescein angiography as the standard against which to test the ability of iris biomicroscopy to demonstrate DI. ${ }^{37}$ The sensitivity of biomicroscopy in detecting DI turned out to be $57 \%$, while the specificity was $94 \%$. The positive predictive value was $93 \%$ and the negative predictive value $50 \%$. Our study proved that biomicroscopy can judge accurately when DI is absent; when it is present, however, there is a high probability that biomicroscopy will be less precise in the detection of iris lesions.

In the present study, in patients without DI, on the other hand, the iris fluorescein angiographic information seems less useful. Sixty nine per cent of our cases had DR but no DI; 35\% were pre-proliferative or proliferative forms of DR. This proportion of false negatives shows that great care is required in the management of the diabetic patient, in whom the fundus can only be explored by ultrasound (because of cataract or vitreous haemorrhage), even if the iris vessels appear normal when examined angiographically. In these patients, when retinal ischaemia is detected, either endophotocoagulation during vitrectomy, or transpupillary retinal photocoagulation after cataract extraction, should be considered mandatory procedures.

So, in answer to our question as to whether DI is an indicator of $\mathrm{DR}$, we can conclude from this study that iris angiography yields valuable information on diabetic ocular microangiopathy, since the presence of DI (especially if serious) is significantly related to the presence of $D R$ in varying degrees of severity. Using iris angiography we were able to detect the eyes with the most serious forms of DR - that is, those most likely to present major complications following surgery. The results of this study are clinically important, as they enable us to consider the presence of retinopathy even when we cannot explore the fundus because of a cataract or vitreous haemorrhage. Fluoroiridography thus appears to be a useful tool when scheduling such eyes for surgery.

However, although the presence of diabetic iridopathy, especially in its severe forms, 
indicates the presence of diabetic retinopathy, the absence of diabetic iridopathy certainly does not exclude the presence of diabetic retinopathy.

Presented in part at the 3rd International Symposium on Ocular Circulation and Neovascularisations, Paris, France, 21-23 May 1992.

1 Apple DJ. Pathology. In: L'Esperance FA, James WA, eds Diabetic retinopathy: clinical evaluation and management. St Louis: Mosby, 1981: 38-57.

2 Blankenship GW. Preoperative iris rubeosis and diabetic vitrectomy results. Ophthalmology 1980; 87: 176-82.

3 Blankenship GW, Machemer R. Pars plana vitrectomy for the management of severe diabetic retinopathy: an analysis of results five years following surgery. Ophthalmology 1978; 85: 553-9.

4 Blankenship GW, Machemer R. Long term diabetic vitrectomy results: report of 10 year follow-up. Ophthalmology 1985; 95: 1-11

5 Eichenbaum DM. Iris neovascularization after pars plana vitrectomy. In: Freeman HM, Hirose T, Schepens CL, eds. Vitreous surgery and advances in fundus diagnosis and treatment. New York: Appleton Century Crofts, 1977: 373-6

6 Rice TA, Michels RG, Maguire MG, Rice EF. The effects of lensectomy on the incidence of iris neovascularization and neovascular glaucoma after vitrectomy for diabetic retinopathy. Am F Ophthalmol 1983; 95: 1-11.

7 Aiello LM, Wand M, Liang G. Neovascular glaucoma and vitreous hemorrhage following cataract surgery in patients vitreous hemorrhage following cataract surgery in pa
with diabetes mellitus. Ophthalmology $1983 ; 90: 814-9$.

8 Apple DJ, Craythorn JM, Olson RJ, Little LE, Lyman JB, Reidy JJ. Anterior segment complications and neovascula glaucoma following implantation of a posterior chambe intraocular lens. Ophthalmology 1984; 91: 403-19.

9 Cunliffe IA, Flanagan DW, George NDL, Aggarwaal RJ, Moore AT. Extracapsular cataract surgery with lens implantation in diabetics with and without proliferative retinopathy. Br f Ophthalmol 1991; 75: 9-12.

10 Jaffe GJ, Burton TC. Progression of nonproliferative diabetic retinopathy following cataract extraction. Arch Ophthalmo 1988; 106: 745-9.

11 Levin ML, Kincaid MC, Eifler CW, Holt JE, Speight JW, O'Connor PS. Effect of cataract surgery and intraocula lenses on diabetic retinopathy. $\mathcal{F}$ Cataract Refract Surg 1988 14: 642-9.

12 Pavese T, Insler MS. Effects of extracapsular cataract extraction with posterior chamber lens implantation on the development of neovascular glaucoma in diabetics. f Cataract Refract Surg 1987; 13: 197-201.

13 Pollack A, Dotan S, Oliver M. The course of diabetic retinopathy following cataract surgery. $\mathrm{Br} \mathcal{F}$ Ophthalmol 1991; 75: 2-8.

14 Pollack A, Dotan S, Oliver M. Progression of diabetic retinopathy after cataract extraction. Brf Ophthalmol 1991; retinopathy

15 Prasad P, Setna PH, Dunne JA. Accelerated ocular neovascularisation in diabetics following posterior chamber len implantation. Brf Ophthalmol 1990; 74: 313-4.

16 Ruiz TS, Saatci OA. Posterior chamber intraocular lens implantation in eyes with inactive and active proliferative diabetic retinopathy. Am f Ophthalmol 1991; 111: 158-62.
17 Sebestyen JG. Intraocular lenses and diabetes mellitus. $A m \mathcal{F}$ Ophthalmol 1986; 101: 425-8.

18 Poliner LS, Christianson DJ, Escoffery RF, Kolker AE Gordon ME. Neovascular glaucoma after intracapsular an extracapsular cataract extraction in diabetic patients. $A m \mathcal{F}$ Ophthalmol 1985; 100: 637-43.

19 Algvere P, Kornacki B. Fluorescein angiography of the iris: a correlation of microangiopathy in the iris and retina. Acta Ophthalmol 1978; 56: 803-16.

20 Bonnet $M$, Jourdain $M$, Francoz-Taillanter N. Correlation neovaisseaux papillaires-rubeosis iridis. $\mathcal{F}$ Fr Ophtalmo $1981 ; 4: 405-10$

21 Brancato R, Bandello F, Carnevalini A, Camesasca F, Meloni $P$. Intraocular lens and diabetes. In: Maumenee AE, Stark WJ, Esente I, eds. Cataract and refractive microsurgery. Milan: Fogliazza, 1987: 133-9.

22 Brancato R, Menchini U, Carnevalini A, Capoferri C. Rubeosis iridis et rétinopathie diabétique. In: Coscas $\mathrm{G}$, ed. La rétinopathie

23. Diabetic Retinopathy Study Research Group. Report 7. A modification of the Airlie House classification of diabetic modification of the Airlie House classification of diabe

24 Brancato R, Menchini U, Carnevalini A. Atlante di iridografia a fluorescenza. Rome: CIC Ed Int Gruppo Ed Medico, 1979 14

25 Kottow M. Fundamentals of angiographic interpretation: the normal anterior segment fluorescein angiogram. In: Kottow M, ed. Anterior segment fluorescein angiography. Baltimore: Williams and Wilkins, 1979: 35-55.

26 Vannas A. Fluorescein angiography of the vessels of the iris in pseudoexfoliation of the lens capsule, capsular glaucoma and some other forms of glaucoma. Acta Ophthalmol Suppl (Kbh) 1969; 105: 1-75.

27 Baggesen $\mathrm{LH}$. Fluorescein angiography of the iris in diabetics and non diabetics. Acta Ophthalmol 1969; 47: 449-60.

28 Demeler V, Sautter H. Irisangiographische untersuchungen bei patienten mit diabetes mellitus. Adv Ophthalmol 1978; 36: 233-44.

29 Ehrenberg M, Brooks W, McCuen II, Schindler RH Machemer R. Rubeosis iridis: preoperative iris fluorescein angiography and periocular steroids. Ophthalmology 1984; 91: $321-5$.

30 Freidburg D. Fluoreszenzangiographie der iris bei diabetiken Klin Monatsbl Augenheilkd 1973; 162: 218-23.

31 Jensen VA, Lundbaek K. Fluorescence angiography of the iris in recent and long term diabetes. Diabetologia 1968; 4: 161-3.

32 Kottow M. Diabetic microangiopathy. In: Kottow M, ed Anterior segment fluorescein angiography. Baltimore: Williams and Wilkins, 1979: 153-76.

33 Laatikainen L. Development and classification of rubeosis iridis in diabetic eye disease. $\mathrm{Br} \mathcal{F}$ Ophthalmol 1979; 63: $150-6$.

34 Lewis ML. Iris fluorescein angiography. Dev Ophthalmol $1981 ; 2: 282-5$.

35 Brancato R, Leoni G, Trabucchi G, Lakidi E, Bulloni M. Contact transscleral irradiation of human chorioretina with continuous wave Nd:YAG laser. Ophthalmic Res 1989; 21: $1-7$

36 Azzolini C, Camesasca F, Brancato $R$. Intraoperative laser photocoagulation. Vitreoret Surg 1991; 3: 1-3.

37 Bandello F, Brancato R, Lattanzio R, Falcomata' B, Malegor A. Biomicroscopy versus fluorescein angiography of the iris in the detection of diabetic iridopathy. Graefes Arch Clin Exp Ophthalmol 1993; 231: 444-8. 